\title{
Study of Reduced Graphene Oxide Preparation by Hummers' Method and Related Characterization
}

\author{
Ning Cao and Yuan Zhang \\ College of Mechanical and Electrical Engineering, China University of Petroleum, Qingdao 266580, China \\ Correspondence should be addressed to Ning Cao; caoning1982@gmail.com
}

Received 13 August 2014; Accepted 25 September 2014

Academic Editor: Liang-Wen Ji

Copyright (C) 2015 N. Cao and Y. Zhang. This is an open access article distributed under the Creative Commons Attribution License, which permits unrestricted use, distribution, and reproduction in any medium, provided the original work is properly cited.

\begin{abstract}
As a novel two-dimensional carbon material, graphene has fine potential applications in the fields of electron transfer agent and supercapacitor material for its excellent electronic and optical property. However, the challenge is to synthesize graphene in a bulk quantity. In this paper, graphite oxide was prepared from natural flake graphite by Hummers' method through liquid oxidization, and the reduced graphene oxide was obtained by chemical reduction of graphene oxide using $\mathrm{NH}_{3} \cdot \mathrm{H}_{2} \mathrm{O}$ aqueous solution and hydrazine hydrate. The raw material graphite, graphite oxide, and reduced graphene oxide were characterized by X-ray diffraction (XRD), attenuated total reflectance-infrared spectroscopy (ATR-IR), and field emission scanning electron microscope (SEM). The results indicated that the distance spacing of graphite oxide was longer than that of graphite and the crystal structure of graphite was changed. The flake graphite was oxidized to graphite oxide and lots of oxygen-containing groups were found in the graphite oxide. In the morphologies of samples, fold structure was found on both the surface and the edge of reduced graphene oxide.
\end{abstract}

\section{Introduction}

Graphene is a novel 2-dimensional material which was first separated from graphite by mechanical stripping method in 2004 [1]. As an allotrope of element carbon, it is a planar sheet of carbon atoms arranged into hexagon [2]. The "thinnest" known material graphene can be used for biosensors [3], transparent electrodes [4], hydrogen storage, composites [5], and high energy supercapacitors [6] for its high optical and electron transparency and excellent mechanical properties [7].

There are many ways to synthesize graphene $[1,8,9]$, such as exfoliation and cleavage, chemical vapor deposition (CVD), thermal decomposition, and electrochemical reduction. Among these preparation methods, solution-based reduction of graphite oxide (GO) is attractive for its easy operation in recent years. It includes three typical steps in this method. They are graphite oxidation, GO aqueous dispersion, and GO reduction. The methods invented by Brodie, Staudenmaier, and Hummers are widely used for graphite oxidation [10]. And Hummers' method is popular for the following reasons. First, $\mathrm{KClO} 3$ was replaced by $\mathrm{KMnO} 4$ as the oxidation agent. In this condition, the byproducts of toxic gas were eliminated and the securities of experiments were improved. Moreover, the oxidation time was shortened, and last it was easy to exfoliate the resulted product in water. In this paper, Hummers' method was employed to prepare GO and reduced GO (rGO) was obtained with the aid of $\mathrm{NH}_{3} \cdot \mathrm{H}_{2} \mathrm{O}$ aqueous and hydrazine hydrate.

\section{Experimental}

2.1. Raw Materials. Flake graphite powder, 98 wt. $\% \mathrm{H}_{2} \mathrm{SO}_{4}$, $\mathrm{KMnO}_{4}, \mathrm{NaNO}_{3}$, deionized water, $\mathrm{NH}_{3} \cdot \mathrm{H}_{2} \mathrm{O}$ aqueous, dilute $\mathrm{HCl}$ aqueous, 30\% $\mathrm{H}_{2} \mathrm{O}_{2}$ aqueous, and $80 \%$ hydrazine hydrate aqueous.

\subsection{Graphene Preparation}

2.2.1. GO Preparation. The mixture of flake graphite/ $\mathrm{NaNO}_{3}$ was prepared in weight ratio of $2: 1$. The mixture was added into a beaker with a certain amount of $98 \mathrm{wt} . \% \mathrm{H}_{2} \mathrm{SO}_{4}$ at $15^{\circ} \mathrm{C}$ and a suspension was obtained. Then, $\mathrm{KMnO}_{4}$ powder which acted as an oxidation agent was gradually added into the suspension with continuous stirring. The weight of the $\mathrm{KMnO}_{4}$ powder is 3 times as much as the one of the mixture. 


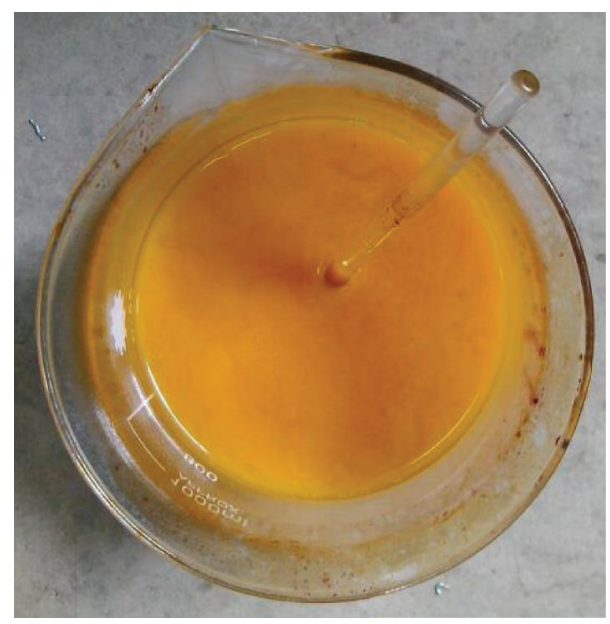

FIGURE 1: Pattern of resulting suspension.

There were 3 steps for the following process. First of all, it is the low temperature reaction. The temperature of the mixture was controlled below $20^{\circ} \mathrm{C}$ for 2 hours; at the same time, the suspension should be stirred continuously. The second step is the mid temperature reaction. The temperature of the mixture was maintained at $35^{\circ} \mathrm{C}$ for 30 minutes after $\mathrm{KMnO}_{4}$ was totally dissolved. Finally, it is the high temperature reaction. A certain amount of deionized water was added into the mixture slowly; therefore a large amount of heat was released when concentrated $\mathrm{H}_{2} \mathrm{SO}_{4}$ was diluted. 15 minutes later, certain amounts of hot water and 30\% $\mathrm{H}_{2} \mathrm{O}_{2}$ aqueous were added into the mixture, respectively, with continuously stirring. As Figure 1 shows, the bright yellow resulted suspension was filtered by the qualitative filter paper when it was still hot, and the solid mixture was washed with dilute $\mathrm{HCl}$ aqueous and distilled water and dried in vacuum oven at $70^{\circ} \mathrm{C}$ for $24 \mathrm{~h}$.

2.2.2. $r$ GO Preparation. $400 \mathrm{mg} \mathrm{GO}$ was dispersed in $400 \mathrm{~mL}$ water by means of 30 minutes' ultrasonic treatment. As a result, a homogeneous brown $\mathrm{GO}$ aqueous suspension was obtained. The $\mathrm{pH}$ of the suspension was adjusted to 10 by dropping $\mathrm{NH}_{3} \cdot \mathrm{H}_{2} \mathrm{O}$. A mount of hydrazine hydrate was added into suspension and heated at $80^{\circ} \mathrm{C}$ for 24 hours, and the weight ratio of hydrazine hydrate and GO was controlled at $10: 7$. A kind of black flocculent substance was gradually precipitated out of the solution. The product was obtained by filtered with the qualitative filter paper. Finally, the resulting black product was washed with methanol and water and dried at $80^{\circ} \mathrm{C}$ for $24 \mathrm{~h}$.

2.3. Characterization of Materials. Crystal features of flake graphite, GO, and rGO were obtained by XRD [10] (AXS cooperation, Germany), with a scan speed of $4^{\circ} / \mathrm{min}$ from 5 to $60^{\circ}$ of $2 \theta$ angles. The layer spacing ( $d$ spacing) could be calculated with the aid of Bragg equation, and the change of diffraction peak could also be observed. The micromorphology of rGO was observed by SEM (JSM-6700F, Japan), with the acceleration voltage from 0.5 to $30 \mathrm{KV}$. Spectra of dried GO and rGO were obtained by Tensor 27 FTIR-ATR (Bruker cooperation, Germany), with the resolution of $4 \mathrm{~cm}^{-1}$ from 3700 to $500 \mathrm{~cm}^{-1}$ of spectral region, and functional groups of $\mathrm{GO}$ and $\mathrm{rGO}$ can be observed.

\section{Result and Discussion}

3.1. X-Ray Diffraction. As shown in Figure 2(a). Flake graphite exhibits a basal diffraction peak (002) at $2 \theta=26.5^{\circ}$ ( $d$ spacing $=0.33630 \mathrm{~nm})$, which is very sharp. There is also a very weak diffraction peak (004) at $2 \theta=54.8^{\circ}$ ( $d$ spacing $=0.16738 \mathrm{~nm})$. The diffraction peak $(004)$ is the second diffraction [11] of the diffraction peak (002) according to layer spatial arrangement rules of microcrystals; thus diffraction peak (004) intensity is much weaker than that of diffraction peak (002). The diffraction peak at about $2 \theta=9.8^{\circ}$ is very typical for GO; no apparent diffraction peak could be found for $\mathrm{rGO}$ in its XRD pattern. This result is similar to that of Tapas Kuila [2], who had already described the structure of GO and rGO by XRD. Figures 2(b) and 2(c) show the enlarged XRD patterns of GO and rGO. Diffraction peak becomes broader in the enlarged pattern of GO at $2 \theta=9.8^{\circ}(d$ spacing $=0.88160)$ and the significant increase in $d$ spacing is believed due to the following reason: oxygen functional groups intercalate in the interlayer of graphite. There is a very weak diffraction peak at $2 \theta=42.3^{\circ}$, which is believed due to the incomplete oxidation. As is shown in Figure 2(c), a very weak and broad diffraction peak can be observed in the XRD pattern of rGO at $2 \theta=25-30^{\circ}$; the diffraction peak of $\mathrm{rGO}$ is so weak that it cannot be visible when drawn together in the XRD pattern with graphite and GO (Figure 2(a)). There is also a weak diffraction peak at $2 \theta=42.3^{\circ}$.

3.2. FTIR-ATR. FTIR-ATR spectra of $\mathrm{GO}$ and $\mathrm{rGO}$ are shown in Figures 3 and 4. Some carbon-oxygen functional groups of $\mathrm{GO}$ are observed in Figure 3, such as $\mathrm{O}-\mathrm{H}, \mathrm{C}=\mathrm{O}, \mathrm{C}-\mathrm{OH}$, and C-O. Characteristic peak $\left(\sim 3464 \mathrm{~cm}^{-1}\right)$ [12] is believed to be attributed to $\mathrm{O}-\mathrm{H}$ stretching of hydroxyl and carboxyl groups, and characteristic peaks of $\mathrm{C}=\mathrm{O}\left(\sim 1639 \mathrm{~cm}^{-1}\right)$, C$\mathrm{OH}\left(\sim 1288 \mathrm{~cm}^{-1}\right)$, and C-O $\left(\sim 1003 \mathrm{~cm}^{-1}\right)$ are also believed to be attributed to carboxylic acid and carbonyl groups [13]. And the characteristic peak at $1493 \mathrm{~cm}^{-1}$ is corresponding to the $\mathrm{C}=\mathrm{C}$ skeletal vibration of unoxidized graphitic domain. These oxygen functional groups indicate that the flake graphite powder has been oxidized to GO. As shown in Figure 4, no obvious peak could be observed, which means that full reduction of GO was made. While carbon-oxygen functional groups all existed, their characteristic peaks are just very weak.

3.3. Scanning Electron Microscope. Figure 5 shows SEM morphologies of $\mathrm{rGO}$ which was dried at $80^{\circ} \mathrm{C}$ for 1 day. As is shown in Figure 5, 2-dimensional material can be observed. Fold structure can be found on both the surface and the edge of rGO powder. They are the typical morphologies of fewlayer rGo [14]. The thickness of rGO may be $10 \mathrm{~nm}$ and it is obviously that rGO layers have fairly large dimension (much larger than $100 \mathrm{~nm}$ ), and rGO retacked together. The reason of the reagglomerate may be the long-time high temperature 


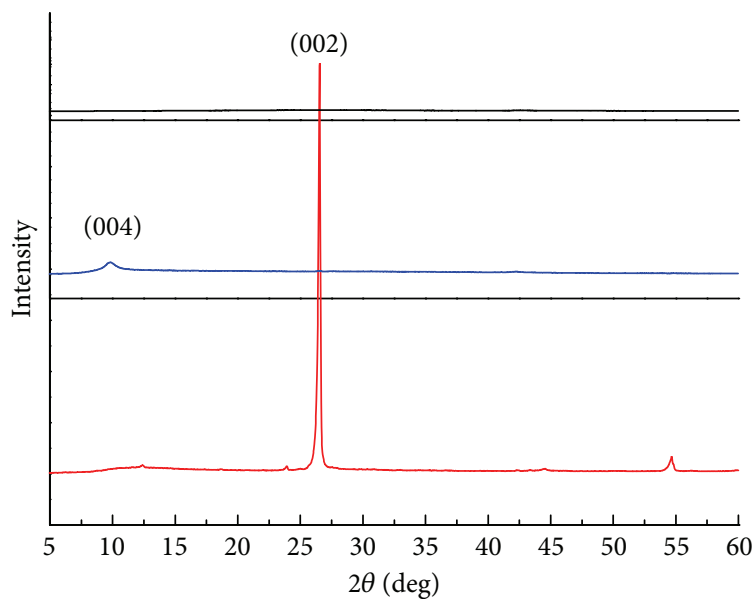

rGO
$-\mathrm{GO}$

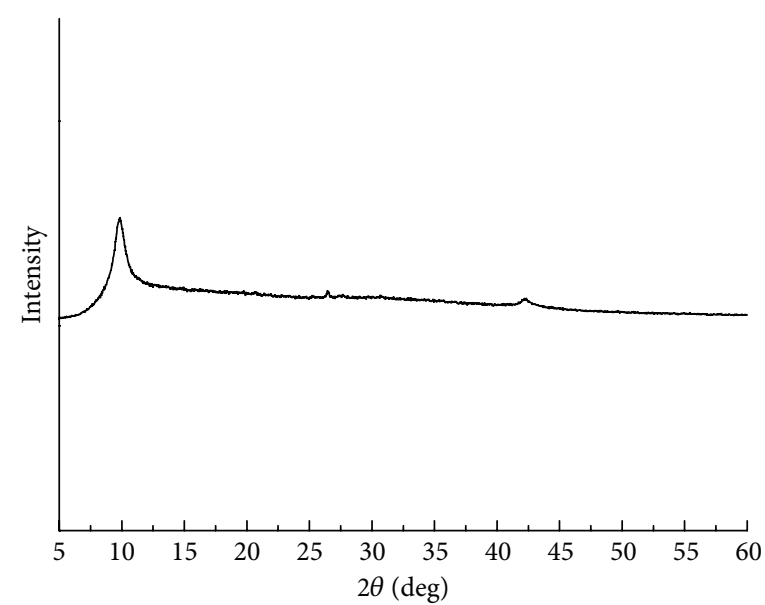

$-\mathrm{GO}$

(a)

(b)

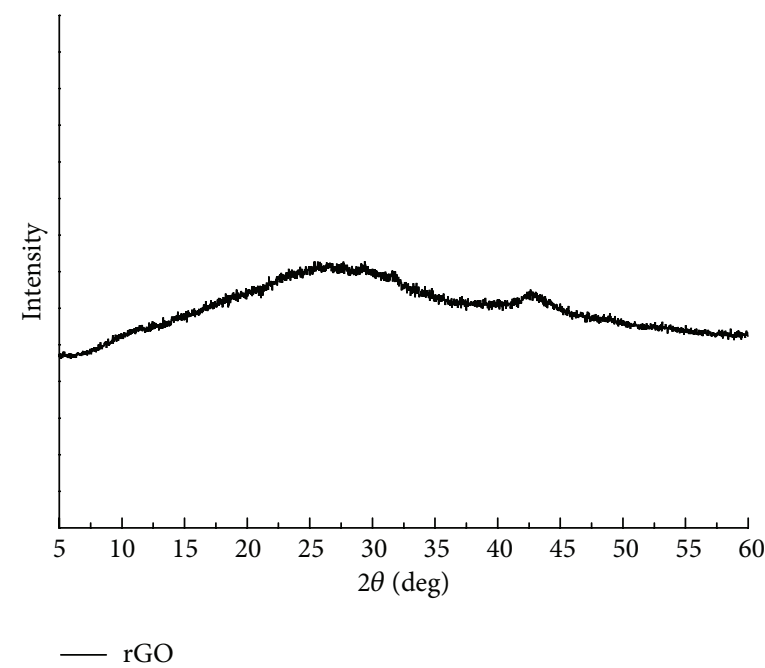

(c)

Figure 2: X-ray diffraction patterns. (a) graphite, GO, and rGO; (b) enlarged view of GO; (c) enlarged view of rGO.

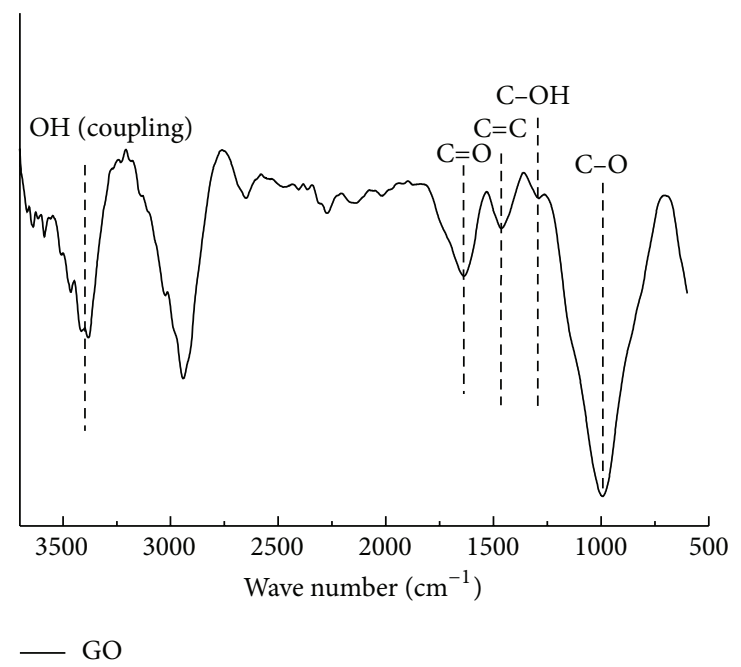

FIGURE 3: FTIR-ATR spectra of GO. 


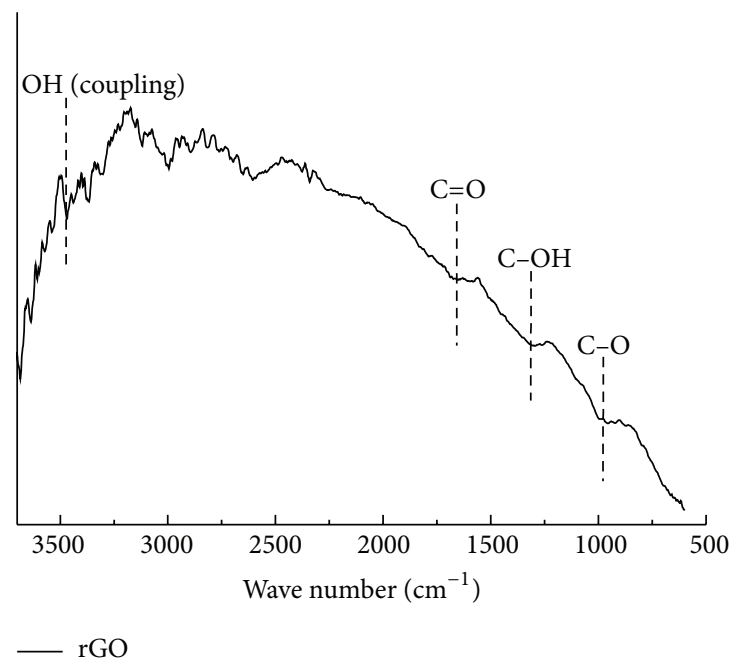

FIGURE 4: FTIR-ATR spectra of rGO.

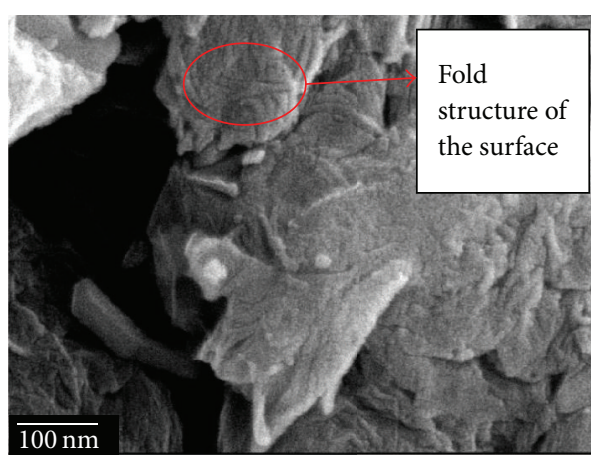

(a)

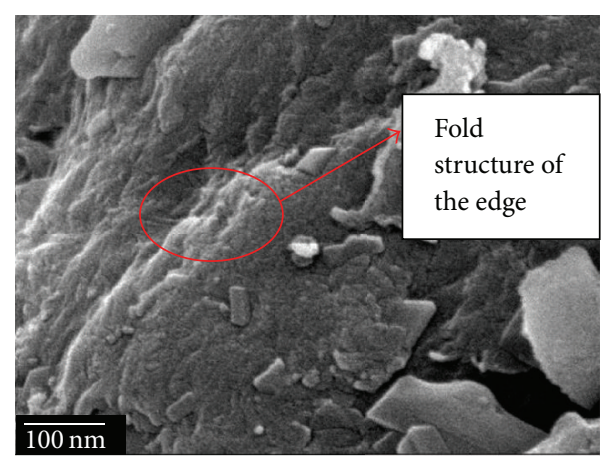

(b)

FIGURE 5: SEM morphology of rGO.

treatment. The morphology of graphite (fold structure) can also be found in Figure 5.

Reaction mechanism of solution-based reduction of graphite oxide will be discussed, to begin with the mechanism of Hummers, though $\mathrm{KMnO}_{4}$ is used as a kind of oxidizing agent. Dreyer et al. [15] believed that the active species was $\mathrm{Mn}_{2} \mathrm{O}_{7}$. The following equation gives the reaction between $\mathrm{KMnO}_{4}$ and $\mathrm{H}_{2} \mathrm{SO}_{4}$ :

$$
\begin{gathered}
\mathrm{KMnO}_{4}+3 \mathrm{H}_{2} \mathrm{SO}_{4} \longrightarrow \mathrm{K}^{+}+\mathrm{MnO}_{3}^{+}+\mathrm{H}_{3} \mathrm{O}^{+}+3 \mathrm{HSO}_{4}^{-} \\
\mathrm{MnO}_{3}^{+}+\mathrm{MnO}_{4}^{-} \longrightarrow \mathrm{Mn}_{2} \mathrm{O}_{7}
\end{gathered}
$$

In low temperature reaction, the edge of graphite was oxidized and intercalated with the aid of oxidizing agent. $-\mathrm{OH}$ was formed during this process. In mid temperature reaction, with the increasing of temperature, the oxidation ability improves furthermore. More oxygen functional groups are formed in this process, and the oxidizing agent penetrates into the internal of graphite layer; therefore this process results in the increasing of $d$ spacing. In the high temperature reaction, concentrated $\mathrm{H}_{2} \mathrm{SO}_{4}$ releases large amount of heat during the process of watering. Force between layers is destroyed and finally the GO could be fully exfoliated to single layers. Secondly, the fully exfoliated GO will be reduced to $\mathrm{rGO}$ with the help of $\mathrm{NH}_{3} \cdot \mathrm{H}_{2} \mathrm{O}$ aqueous and hydrazine hydrate.

The mechanism of solution-based reduction of GO is quite different with that of the traditional CVD ones. The formation of graphene on bulk metal through CVD includes three steps $[1,16,17]$. First, a hydrocarbon could be dissociated through dehydrogenation; second, the carbon species diffuse and dissolve into the bulk metal at the growth temperature; the reason why transition metals could serve as an electron acceptor is because of the empty dshell; third, carbon species precipitate out of the bulk metal onto the metal surface upon the rapid quenching, start the segregation process, and build up honeycomb lattice because the solubility decreases during the cooling process.

\section{Conclusion}

GO was prepared by Hummers' method and rGO was prepared with the aid of $\mathrm{NH}_{3} \cdot \mathrm{H}_{2} \mathrm{O}$ aqueous and hydrazine hydrate successfully. The characterization results indicate that 
the layer spacing of graphite oxide was longer than that of graphite. The crystal structure of graphite was changed. Graphite was oxidized to GO and lots of oxygen-containing groups were found in the GO. The typical fold morphologies were found on both the surface and the edge of rGO.

Compared with the traditional CVD method, Hummers' method can synthesize GO in large scale, then rGO can be prepared with the help of reduced agent, and this process costs a little. Meanwhile, the prepared GO is dispersed easily in solution. In this case, the modification of the GO is easy and it is suitable for GO application in composites and energy storage devices.

\section{Conflict of Interests}

The authors declare that there is no conflict of interests regarding the publishing of this paper.

\section{Acknowledgments}

The authors acknowledge funding from the National Natural Science Foundation of China (no. 51302320) and the Fundamental Research Funds for the Central Universities (no. 14CX05094A).

\section{References}

[1] V. Singh, D. Joung, L. Zhai, S. Das, S. I. Khondaker, and S. Seal, "Graphene based materials: past, present and future," Progress in Materials Science, vol. 56, no. 8, pp. 1178-1271, 2011.

[2] M. I. Katsnelson, "Graphene: carbon in two dimensions," Materials Today, vol. 10, no. 1-2, pp. 20-27, 2007.

[3] T. Kuila, S. Bose, P. Khanra, A. K. Mishra, N. H. Kim, and J. H. Lee, "Recent advances in graphene-based biosensors," Biosensors and Bioelectronics, vol. 26, no. 12, pp. 4637-4648, 2011.

[4] G. Eda, G. Fanchini, and M. Chhowalla, "Large-area ultrathin films of reduced graphene oxide as a transparent and flexible electronic material," Nature Nanotechnology, vol. 3, no. 5, pp. 270-274, 2008.

[5] T. Kuilla, S. Bhadra, D. Yao, N. H. Kim, S. Bose, and J. H. Lee, "Recent advances in graphene based polymer composites," Progress in Polymer Science, vol. 35, no. 11, pp. 1350-1375, 2010.

[6] B. G. Choi, M. Yang, W. H. Hong, J. W. Choi, and Y. S. Huh, “3D macroporous graphene frameworks for supercapacitors with high energy and power densities," ACS Nano, vol. 6, no. 5, pp. 4020-4028, 2012.

[7] Y. Zhu, S. Murali, W. Cai et al., "Graphene and graphene oxide: synthesis, properties, and applications," Advanced Materials, vol. 22, no. 35, pp. 3906-3924, 2010.

[8] T. Kuila, S. Bose, A. K. Mishra, P. Khanra, N. H. Kim, and J. H. Lee, "Chemical functionalization of graphene and its applications," Progress in Materials Science, vol. 57, no. 7, pp. 1061-1105, 2012.

[9] S. Y. Toh, K. S. Loh, S. K. Kamarudin, and W. R. Wan Daud, "Graphene production via electrochemical reduction of graphene oxide: synthesis and characterisation," Chemical Engineering Journal, vol. 251, pp. 422-434, 2014.

[10] C. Bao, L. Song, W. Xing et al., "Preparation of graphene by pressurized oxidation and multiplex reduction and its polymer nanocomposites by masterbatch-based melt blending," Journal of Materials Chemistry, vol. 22, no. 13, pp. 6088-6096, 2012.

[11] A. Bagri, C. Mattevi, M. Acik, Y. J. Chabal, M. Chhowalla, and V. B. Shenoy, "Structural evolution during the reduction of chemically derived graphene oxide," Nature Chemistry, vol. 2, no. 7, pp. 581-587, 2010.

[12] Y. Jin, S. Sun, and X. Qi National Defense Industry Press, 2008, (Chinese).

[13] Q. Deng, L. Liu, and H. Deng, Spectrum Analysis Tutorial, Science Press, 2007, (Chinese).

[14] F. Hong, L. Zhou, and Y. Huang, "Synthesis and characterization of graphene by improved hummers method," Chemical \& Biomolecular Engineering, vol. 29, pp. 31-33, 2012 (Chinese).

[15] D. R. Dreyer, S. Park, C. W. Bielawski, and R. S. Ruoff, "The chemistry of graphene oxide," Chemical Society Reviews, vol. 39, no. 1, pp. 228-240, 2010.

[16] Y. Kai, F. Lei, L. P. Hai, and F. L. Zhong, "Designed CVD growth of graphene via process engineering," Accounts of Chemical Research, vol. 46, pp. 2263-2274, 2013.

[17] C.-M. Seah, S.-P. Chai, and A. R. Mohamed, "Mechanisms of graphene growth by chemical vapour deposition on transition metals," Carbon, vol. 70, pp. 1-21, 2014. 

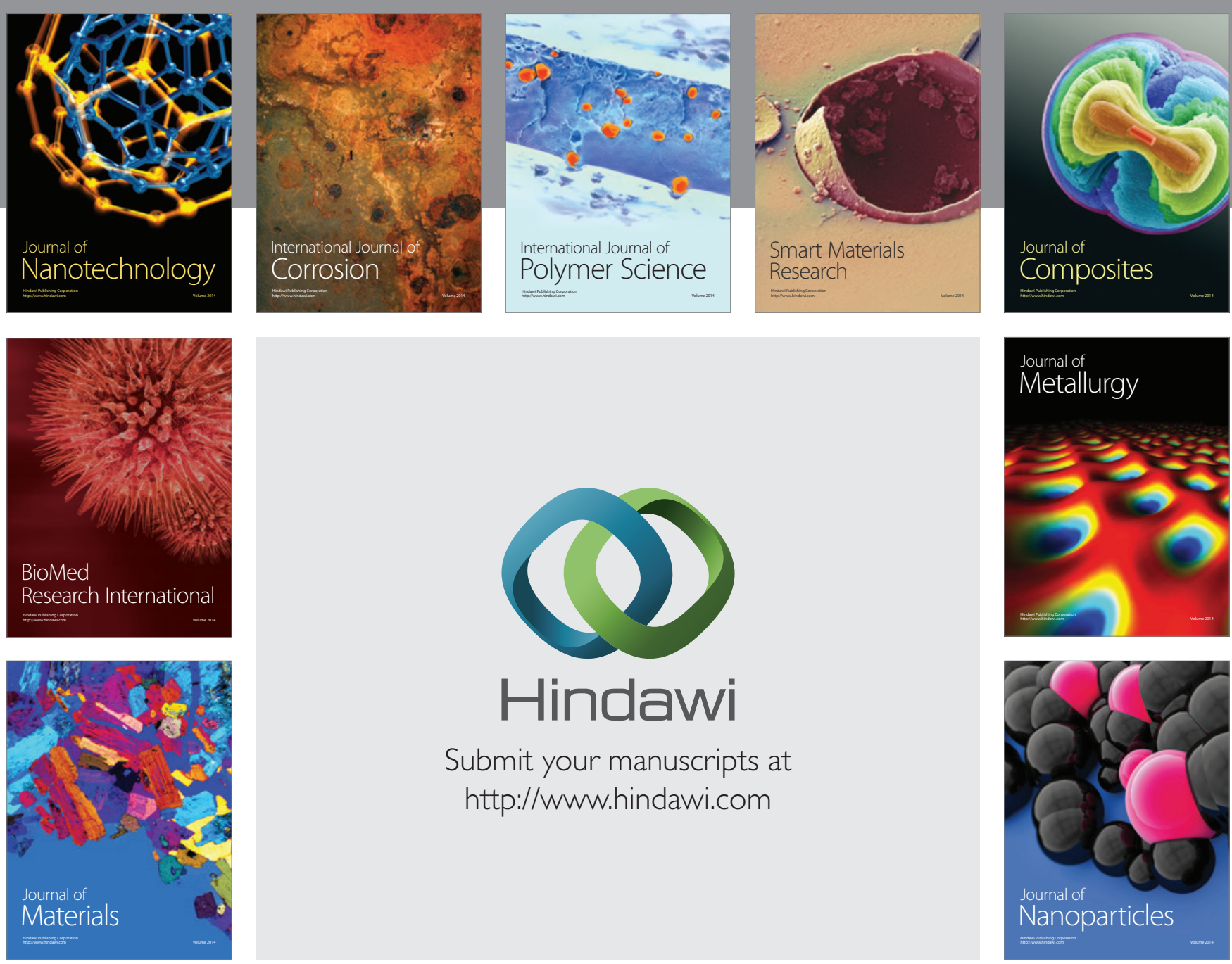

Submit your manuscripts at http://www.hindawi.com
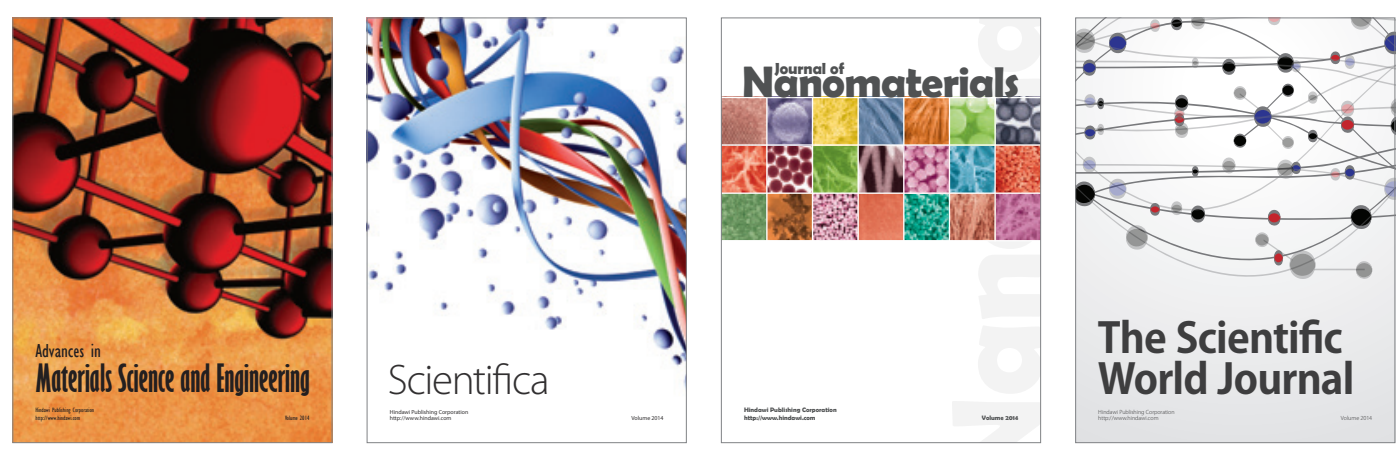

\section{The Scientific World Journal}
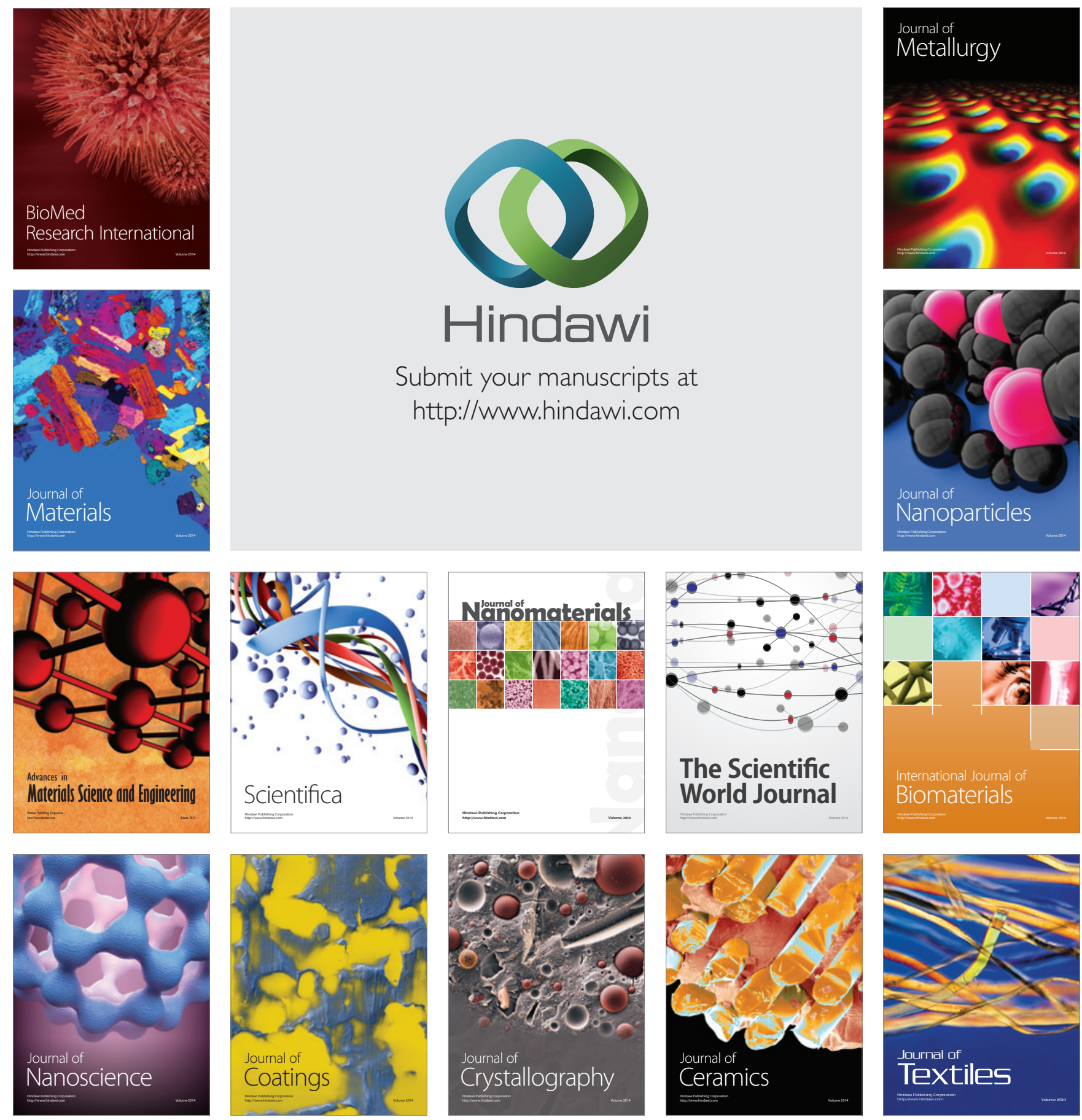